\title{
The role of cardiopulmonary exercise testing (CPET) in predicting mortality and morbidity in people with congenital heart disease: a systematic review and meta- analysis (Protocol)
}

Curtis A. Wadey ${ }^{1}$, Max E. Weston ${ }^{1}$, Dan Mihai Dorobantu', Rod S. Taylor ${ }^{2}$, Guido E. Pieles ${ }^{1,3,4}$, Alan R. Barker ${ }^{1}$ and Craig A. Williams ${ }^{1 *}(\mathbb{D}$

\begin{abstract}
Background: Numerous studies have measured the prognostic associations between cardiorespiratory fitness and patient outcomes in congenital heart disease, but no systematic review has assessed these associations for all types of congenital heart disease. It is therefore a timely opportunity to syntheses all available data using a systematic review methodology. The aim of this study is to detail the protocol for a systematic review and meta-analysis.

Objectives: Within this paper we have developed a protocol for a prognostic factors systematic review and metaanalysis, to assess the role of cardiopulmonary exercise testing/cardiorespiratory fitness, in the prognosis of mortality and morbidity in congenital heart disease.
\end{abstract}

Methods: We have outlined, in detail, the process for this systematic review using the latest accepted methodological guidelines for prognostic factors research, such as the PICOTS system, CHARMS-PF data extraction, QUIPS risk of bias assessments and the prognostic GRADE guidelines (see list of abbreviations).

Conclusion: The implications of this review will aid future treatments, interventions and individual patient risk prediction. The publication of this protocol aims to improve scientific rigour by ensuring transparency in the systematic review and meta-analysis process.

Keywords: Cardiorespiratory fitness, Exercise test, Mortality, Morbidity, Prognosis, Congenital heart, Systematic review

\footnotetext{
* Correspondence: c.a.williams@exeter.ac.uk

${ }^{1}$ Children's Health and Exercise Research Centre, University of Exeter, St Luke's Campus, Heavitree Road, Exeter, Devon EX1 2LU, UK

Full list of author information is available at the end of the article
}

(c) The Author(s). 2020 Open Access This article is licensed under a Creative Commons Attribution 4.0 International License, which permits use, sharing, adaptation, distribution and reproduction in any medium or format, as long as you give appropriate credit to the original author(s) and the source, provide a link to the Creative Commons licence, and indicate if changes were made. The images or other third party material in this article are included in the article's Creative Commons licence, unless indicated otherwise in a credit line to the material. If material is not included in the article's Creative Commons licence and your intended use is not permitted by statutory regulation or exceeds the permitted use, you will need to obtain permission directly from the copyright holder. To view a copy of this licence, visit http://creativecommons.org/licenses/by/4.0/. The Creative Commons Public Domain Dedication waiver (http://creativecommons.org/publicdomain/zero/1.0/) applies to the data made available in this article, unless otherwise stated in a credit line to the data. 


\section{Introduction}

Congenital heart disease (ConHD) is a structural abnormality of the heart or intrathoracic vessels [1], occurring in approximately $1 \%$ of all births worldwide $[2,3]$. These defects are inclusive of a broad spectrum of over 18 different types of cardiac pathologies [4-6]. Longterm survival is reduced in this patient population [7] with repair status and severity of the lesion being risk factors for excess mortality [8]. However, as a result of improved medical care, survival over recent decades has increased, leading to the highest proportion of ConHD deaths now occurring in geriatrics $[7,9]$.

Cardiorespiratory fitness (CRF) is overall lower in patients with ConHD compared to healthy controls, it is also highly heterogeneous both within and between individual ConHD diagnoses, with the more severe conditions presenting with lower levels of fitness [10]. This is important as several studies have reported significant associations between CRF and mortality and morbidity in ConHD [11-15].

Cardiopulmonary exercise testing (CPET) comprises a known exercise stimulus with the simultaneous measurement of pulmonary gas exchange, heart rate and blood pressure. Analysis of CPET data can quantify various measures of CRF e.g., peak exercise capacity, physiological thresholds, efficiency slopes and other physiological responses to the demands of the exercise stimulus [16]. It has been recommended that CPET is used routinely in the care of congenital heart disease patients in order to evaluate and track disease severity [10].

While there has been a number of prognostic factor studies on this topic, there has been no previous systematic review to synthesise all available data on cardiorespiratory fitness and prognosis in congenital heart disease. We aim to conduct this review to inform health care policy and to highlight future avenues of research.

\section{Objectives}

This review will aim to assess the role of cardiopulmonary exercise testing (CPET) in the prognosis of mortality and morbidity in individual congenital heart disease lesions.

\section{Methods}

This protocol has been submitted in the international prospective register of systematic reviews (PROSPERO; CRD42020186518).

\section{Types of participants}

Any individual with a confirmed diagnosis of structural ConHD will be included, regardless of severity, age, sex and previous medical intervention. Exceptions include patients who have a circulatory assist device in situ or are post heart transplant, as they no longer reflect
ConHD physiology. We will also exclude patients with cardiac diseases, such as acquired heart disease (valvular and ischemic coronary disease), infective heart disease or other types of inherited cardiac conditions (arrhythmia, cardiomyopathy etc.). If mixed populations are reported (e.g. cardiomyopathy separate to congenital heart disease population), firstly we will consider subgroup analyses, secondly contact the authors for data and if we cannot resolve inclusion by these steps, we will exclude the study if ConHD is not represented in at least $70 \%$ of the sample.

\section{Types of studies}

We will include any study type that addresses the research question (CPET \& outcome), this will be inclusive of, but not limited to cross-sectional and longitudinal studies (prospective \& retrospective). Individual case reports, review papers, editorials and conference abstracts with no subsequent peer reviewed full-text paper will be excluded. We will exclude any paper that is not written in English, unless provided with a translated manuscript by the authors.

\section{Methods of CPET and interpretation}

Any treadmill and/or cycle ergometer protocol that directly measures pulmonary gas exchange will be included. Exclusion criteria include any exercise protocols that do not directly measure gas exchange or fitness, such as the 6 min walk test. Expected parameters reported are maximal oxygen uptake ( $\dot{\mathrm{VO}}_{2}$ max), oxygen uptake efficiency slope, ventilatory equivalents for carbon dioxide slope ( $\dot{\mathrm{VE}} / \mathrm{VCO}_{2}$ slope), exercise oscillatory ventilation and the gas exchange threshold. Inclusion criteria will not be limited to the expected parameters, any parameter that can be collected or computed (e.g. blood pressure and circulatory power) from a formal CPET shall be reviewed as an 'index prognostic factor'. We anticipate a variety of different 'comparator prognostic factors' (covariates) to be reported, however, this will not form the basis for our inclusion criteria.

\section{Timing and setting}

There will be no limits on timing and the setting of prognostic factors (CPET results) within the review. The timing of the CPET can be at any time point (pre/postoperative or apart of routine follow up); traditionally performed in a secondary or tertiary care provider.

\section{Outcomes}

\section{Primary outcomes}

- All-cause mortality or cardiac-related mortality

- Transplant or ventricular assist device

- Hospitalisation for a cardiac cause 
- Major Adverse Cardiovascular Event (composite of cardiac death, transplant/ventricular assist device, hospitalisation for cardiac cause).

The primary outcomes listed above will form the inclusion criteria for the review. Included studies will then be checked to see if they contribute further data to our secondary outcomes of interest.

\section{Secondary outcomes}

- Systolic and/or diastolic cardiac function

- Serious adverse events during CPET

- Cardiac intervention or re-intervention (surgical, transcatheter or hybrid)

- Other morbidity (i.e. disease specific outcome)

\section{Search methods}

We will search the following bibliographic databases:

Allied and Complementary Medicine Database, CINA

$\mathrm{HL}^{\circ}$ Complete and SPORTDiscus via EBSCO. Medline and Embase will be searched via Ovid. We will also search Web of Science (Thomson Reuters) and the Cochrane Central Register of Controlled Trials.

We will also backward citation chase (search the reference list of relevant reviews and editorials), to find any studies that may have been missed by the electronic searches. We will also forward citation chase for any paper that meets the inclusion criteria.

Our search terms will encompass prognosis (mortality, morbidity, event free survival etc.) with congenital heart disease (Fontan, Tetralogy of Fallot etc.) and CPET variables (maximal oxygen uptake $\dot{\mathrm{VO}}_{2}$ max, Peak $\mathrm{VO}_{2}$, $\dot{\mathrm{VE}} /$ $\dot{\mathrm{V} C O}{ }_{2}$ slope etc.).The search strategy (Appendix) will be adapted for use with the other bibliographic databases. The search will be performed in July 2020, with no lower limit for publication date. A repeat search will be performed if screening, data analysis and publication requires a period longer than 12 months.

\section{Data collection and analysis}

After the deduplication of the searches, two independent reviewers using Covidence ${ }^{\circ}$ (Veritas Health Innovation Ltd., Melbourne, Australia) will screen the titles and abstracts. The full text of potentially eligible studies will be retrieved and assessed for eligibility again independently by two reviewers. Disagreements will be resolved by consensus discussion, if the two review authors cannot resolve the disagreement, a third author will arbitrate. If there are missing data we will contact authors for clarification. We will include all studies that meet our inclusion/exclusion criteria; record the process using the Preferred reporting items for systematic review and meta-analysis (PRISMA) guidelines, and present a PRIS
MA flow diagram and 'Characteristics of excluded studies' table $[17,18]$.

In order to extract data from the eligible studies, a piloted form will be used. Data will be extracted by two reviewers independently and discrepancies solved within the review team. Where possible, missing data will be requested from the study authors. The checklist 'critical appraisal and data extraction for systematic reviews of prediction modelling and prognostic factors studies' (CHARMS-PF) will be used for data extraction, as it facilitates comprehensive extraction of the following: source of the data, participants, outcomes to be predicted, prognostic factors (types and timing), sample size, missing data, analysis, results and interpretation $[19,20]$. Where studies have not optimally reported key study data, such as the analysis method of their CPET data, then we will contact the corresponding authors of the text and ask for clarification.

\section{Assessment of risk of bias}

Two review authors will independently assess the quality of the studies (risk of bias) using the Cochrane adopted Quality in Prognosis Studies tool (QUIPS) [21]. This tool supports a systematic appraisal of bias in studies, it interrogates 6 domains of prognostic factor studies:

1. Study Participation

2. Study Attrition

3. Prognostic Factors

4. Outcome Measurement

5. Study Confounding and

6. Statistical analysis

\section{Dealing with missing data}

Where necessary we will contact authors or principle investigators to verify key study characteristics or obtain missing data. If necessary we will convert medians and interquartile ranges to means and standard deviations using a validated Excel $\odot$ calculator [22]. Furthermore, we will obtain unadjusted hazard ratios estimates where they have not been reported, using established methods [23, 24].

\section{Data synthesis}

We will undertake meta-analyses only where this is meaningful, i.e. if the participants, intervention, outcomes and the underlying clinical question are similar enough for pooling to yield interpretable results. If there is sufficient data for meta-analyses to be undertaken, unadjusted (univariate) and adjusted (multivariate) estimates will be analysed separately, in order to find a 'genuine' prognostic factor [20]. Where studies have controlled for different covariates in the multivariate analyses, we will transparently present what covariates each study controlled for using footnotes. Furthermore, 
hazard ratios, odds ratios and risk ratios will also be meta-analysed separately [20].

Following the recommendations of Riley and colleagues [20], we will pool data using a random-effects meta-analysis due to the high probability of statistical heterogeneity, which will be further explored using subgroup analyses and meta-regression. However, we may also report the fixed-effect pooled estimate and 95\% confidence interval only as a sensitivity analysis because of the tendency of smaller trials, which are more susceptible to publication bias, to be over-weighted with a random-effects analysis.

We will also assess publication bias using visual inspection of funnel plots and the use of the Peter's and Debray's tests for risk, odds and hazard ratios $[25,26]$.

We will provide a narrative synthesis of the findings, there will be a section on each fitness parameter reported (i.e., $\dot{\mathrm{VO}}_{2} \max$ ) and its prognostic role in each individual congenital heart disease lesion. If the data are available and sufficient, CPET variables will be combined to provide appropriate prognostic 'cut-offs'. If metaanalysis is not undertaken we will follow the 'Synthesis without meta-analysis' (SWiM) guidelines to ensure transparency of our methods [27]. We will also use the SWiM guidelines to enhance any written synthesis of meta-analysed data.

\section{Grades of recommendation assessment development and evaluation (GRADE)}

GRADE facilitates a standardised approach when assessing the certainty of evidence, it is based on several domains such as methodological limitations, inconsistency, indirectness and impression [28]. Two review authors will independently undertake GRADE, and where there are discrepancies a third experienced author will arbitrate. Authors will follow the latest GRADE guidelines for assessing evidence in prognostic factor studies by Foroutan et al. [28]. Conducting GRADE should make our findings more understandable to lay members such as policy makers.

\section{Subgroup analysis and investigation of heterogeneity}

Each CPET variable reported will be a subgroup for the analyses along with each individual type of ConHD lesion. If it is possible to pool ten or more studies in a meta-analysis, we will explore heterogeneity using univariate meta-regression in Stata $\odot$. Potential sources of heterogeneity include:

- Age

- Percentage male/female

- Body mass index

- Percentage of population taking chronotropic medication
- Year of study

- Location of study (continent)

- Severity of ConHD [Hoffman criteria [29]]

- Associated pulmonary pathology such as pulmonary hypertension

- Sample size

- Length of follow up

- Risk of bias

- Number comparison prognostic factors

- Timing of the prognostic factors (routine care vs. pre-operative etc.)

\section{Sensitivity analysis}

We will undertake sensitivity analyses comparing: random effects vs. fixed effects meta-analysis and all studies vs. exclusion of high risk of bias studies.

\section{Conclusion}

The publication of systematic review protocols increases the transparency and robustness of the systematic review process as any unplanned changes between the protocol and the review will be explicit and will have to be justified. This protocol for the role of cardiopulmonary exercise testing (CPET) in predicting mortality and morbidity in people with congenital heart disease: a systematic review and meta-analysis' outlines our planned review and the robust systematic process that will be undertaken.

\section{Appendix}

\section{Search strategy}

Medline.

1\# prognosis/ or exp. disease-free survival/.

2\# (Prognosis or forecast or prospect or prediction or projection or mortality or morbidity or transplant or event free survival or hospitali?ation).ti,ab,kw.

3\# exp. heart defects, congenital/ or exp. vascular malformations/ or exp. heart valve diseases/ or exp. ventricular outflow obstruction/ or exp. cardiovascular abnormalities/.

4\# (Congenital heart disease or Congenital heart diseases or congenital heart defect or congenital heart or ConHD or CHD or cyanotic heart disease or cyanotic heart disease* or GUCH).ti,ab,kw.

5\# (atrial septal defect or asd or septal defect or patent ductus arteriosus or pda or bicuspid aortic valve or bav or atresia or surgical repair or d-transposition or transposition great arteries or transposition of great arteries or tga or crisscross heart* or mustard or arterial switch or atrial switch or tetralogy of fallot or fontan or atriopulmonary connection or intracardiac total cavopulmonary connection or pulmonary atresia or absent pulmonary valve or hypoplastic heart or hypoplastic right heart or atresia or tricuspid atresia or pulmonary atresia or Ebstein anomaly or hypoplastic left heart or 
aortic atresia or mitral atresia or single ventricle or systemic right ventricle or sv or dorv or double outlet right ventricle or truncus arteriosus or total anomalous pulmonary venous connection or pulmonary stenosis or double outlet left ventricle or acyanotic lesions or atrioventricular septal defect or avsd or vsd or ventricular septal defect or aortic stenosis or pulmonary stenosis or coarctation or aortic incompetence).ti,ab,kw.

6\# exp. exercise test/ or exp. physical endurance/ or exp. anaerobic threshold/ or exp. exercise tolerance/ or exp. physical fitness/ or exp. cardiorespiratory fitness/.

7\# (Cardiopulmonary exercise test or CPET or Exercise test or exercise tolerance test or fitness test or cycle ergometry or treadmill ergometry or maximal oxygen consumption or $\dot{\mathrm{VO}}_{2}$ or $\mathrm{VO}_{2}$ max or $\mathrm{VO}_{2}$ peak or peak $\mathrm{VO}_{2}$ or $\mathrm{O}_{2}$ pulse or ventilatory equivalent" or OUES or oxygen uptake efficiency slope or petco 2 or exercise oscillatory ventilation or oxygen uptake kinetics or end tidal carbon or cardiorespiratory fitness or aerobic power or exercise capacity or ventilatory threshold or VAT or GET or gas exchange threshold or anaerobic threshold).ti,ab,kw.

(\#1 or \#2 AND \#3 or \#4 or \#5 AND \#6 or \#7)

\section{Abbreviations}

ConHD: Congenital heart disease; CRF: Cardiorespiratory fitness; CPET: Cardiopulmonary exercise testing; $\dot{\mathrm{V}}_{2}$ max: Maximal oxygen uptake; $\dot{V} E \mathrm{NCO}_{2}$ : Ventilatory equivalent for carbon dioxide slope; PICOTS: Population, Index prognostic factor, Comparator prognostic factors, Outcome, Timing, Setting; QUIPS: Quality in Prognosis Studies; PRISMA: Preferred reporting items for systematic review and meta-analysis; CHARMS-PF: Critical appraisal and data extraction for systematic reviews of prediction modelling and prognostic factors studies'; SWiM: Synthesis without meta-analysis';

GRADE: Grades of recommendation assessment development and evaluation

\section{Acknowledgements}

We would like to thank information specialist Simon Briscoe who helped edit the search strategy and search terms.

\section{Authors' contributions}

CA Wadey designed (the study protocol and search strategy), wrote, edited, and finalised the manuscript. MEW and DMD edited the search terms and manuscript. RST reviewed the systematic methods and statistical plan. GEP gave special insight into the literature and population with congenital heart disease. ARB supervised and reviewed the manuscript. CA Williams supervised, edited and reviewed the manuscript. All authors read and approved the final manuscript.

\section{Funding}

CA Wadey is funded by an industrial PhD scholarship by Canon Medical Systems UK Ltd. and the University of Exeter. MEW is funded by a PhD scholarship from the QUEX institute and DMD is funded by the Medical Research Council. The funding bodies had no influence over the design or production of this protocol. The publication costs for this paper were paid for by the University of Exeter.

\section{Availability of data and materials}

Not applicable.

Ethics approval and consent to participate

Not applicable.

\section{Consent for publication}

Not applicable.

\section{Competing interests}

GEP is lead researcher in a contractual research partnership between the University of Bristol and Canon Medical Systems UK Ltd. investigating cardiac function during exercise in children. All other authors declare that they have no competing interests.

\section{Author details}

'Children's Health and Exercise Research Centre, University of Exeter, St Luke's Campus, Heavitree Road, Exeter, Devon EX1 2LU, UK. ${ }^{2}$ Institute of Health and Wellbeing, University of Glasgow, Glasgow, UK. ${ }^{3}$ School of Population Health Sciences and School of Translational Health Sciences, University of Bristol, Bristol, UK. ${ }^{4}$ Congenital Heart Unit, Bristol Royal Hospital for Children and Heart Institute, Bristol, UK.

Received: 29 May 2020 Accepted: 28 July 2020

Published online: 07 September 2020

\section{References}

1. Mitchell SC, Korones SB, Berendes HW. Congenital heart disease in 56,109 births. Incidence and natural history. Circulation. 1971;43(3):323-32.

2. Van Der Linde D, Konings EEM, Slager MA, Witsenburg M, Helbing WA, Takkenberg JJM, et al. Birth prevalence of congenital heart disease worldwide: a systematic review and meta-analysis. J Am Coll Cardiol. 2011;58(21):2241-7.

3. Liu Y, Chen S, Zühlke L, Black GC, Choy MK, Li N, et al. Global birth prevalence of congenital heart defects 1970-2017: updated systematic review and meta-analysis of 260 studies. Int J Epidemiol. 2019;48(2):455-63.

4. Sommer RJ, Hijazi ZM, Rhodes JF. Pathophysiology of congenital heart disease in the adult: part III: complex congenital heart disease. Circulation. 2008;117(10):1340-50

5. Rhodes JF, Hijazi ZM, Sommer RJ. Pathophysiology of congenital heart disease in the adult, part II: simple obstructive lesions. Circulation. 2008; 117(9):1228-37.

6. Sommer RJ, Hijazi ZM, Rhodes JF. Pathophysiology of congenital heart disease in the adult part l: shunt lesions. Circulation. 2008;117(8):1090-9.

7. Best KE, Rankin J. Long-term survival of individuals born with congenital heart disease: a systematic review and meta-analysis. J Am Heart Assoc. 2016:5(6):1-16.

8. Oliver JM, Gallego P, Gonzalez AE, Garcia-Hamilton D, Avila P, Yotti R, et al. Risk factors for excess mortality in adults with congenital heart diseases. Eur Heart J. 2017;38(16):1233-41.

9. Khairy P, lonescu-Ittu R, MacKie AS, Abrahamowicz M, Pilote L, Marelli AJ. Changing mortality in congenital heart disease. J Am Coll Cardiol. 2010; 56(14):1149-57.

10. Amedro P, Gavotto A, Guillaumont S, Bertet H, Vincenti M, De La Villeon G, et al. Cardiopulmonary fitness in children with congenital heart diseases versus healthy children. Heart. 2018;104(12):1026-36.

11. Giardini A, Hager A, Lammers AE, Derrick G, Müller J, Diller GP, et al. Ventilatory efficiency and aerobic capacity predict event-free survival in adults with atrial repair for complete transposition of the great arteries. J Am Coll Cardiol. 2009;53(17):1548-55.

12. Cunningham JW, Nathan AS, Rhodes J, Shafer K, Landzberg MJ, Opotowsky AR. Decline in peak oxygen consumption over time predicts death or transplantation in adults with a Fontan circulation. Am Heart J. 2017;189:184-92.

13. Tsai YJ, Li MH, Tsai WJ, Tuan SH, Liao TY, Lin KL. Oxygen uptake efficiency slope and peak oxygen consumption predict prognosis in children with tetralogy of Fallot. Eur J Prev Cardiol. 2016;23(10):1045-50.

14. Müller J, Hager A, Diller GP, Derrick G, Buys R, Dubowy KO, et al. Peak oxygen uptake, ventilatory efficiency and QRS-duration predict event free survival in patients late after surgical repair of tetralogy of Fallot. Int J Cardiol. 2015;196:158-64

15. Udholm S, Aldweib N, Hjortdal VE, Veldtman GR. Prognostic power of cardiopulmonary exercise testing in Fontan patients: a systematic review. Open Hear. 2018;5(1):e000812.

16. Wasserman K, Hansen J, Sue D, Stringer W, Whipp B. Principles of exercise testing and interpretation. Including pathophysiology and clinical applications. 4th ed. Philadelphia: Lippincott Williams and Wilkins; 2005.

17. Shamseer L, Moher D, Clarke M, Ghersi D, Liberati A, Petticrew M, et al. Preferred reporting items for systematic review and meta-analysis protocols (prisma-p) 2015: elaboration and explanation. BMJ. 2015;349(January):1-25.

18. Liberati A, Altman DG, Tetzlaff J, Mulrow C, Gøtzsche PC, loannidis JPA, et al. The PRISMA statement for reporting systematic reviews and meta-analyses 
of studies that evaluate healthcare interventions: explanation and elaboration. BMJ. 2009;21:339.

19. Moons KGM, de Groot JAH, Bouwmeester W, Vergouwe Y, Mallett S, Altman DG, et al. Critical Appraisal and Data Extraction for Systematic Reviews of Prediction Modelling Studies: The CHARMS Checklist. PLoS Med. 2014;11(10): e1001744.

20. Riley RD, Moons KGM, Snell KIE, Ensor J, Hooft L, Altman DG, et al. A guide to systematic review and meta-analysis of prognostic factor studies. BMJ. 2019;30:364

21. Hayden JA, van der Windt DA, Cartwright JL, Côté P, Bombardier C. Assessing bias in studies of prognostic factors. Ann Intern Med. 2013;158(4):280-6.

22. Wan $X$, Wang W, Liu J, Tong T. Estimating the sample mean and standard deviation from the sample size, median, range and/or interquartile range. BMC Med Res Methodol. 2014;14(1):1-13.

23. Parmar MKB, Torri V, Stewart L. Extracting summary statistics to perform meta-analyses of the published literature for survival endpoints. Stat Med. 1998:17(24):2815-34

24. Tierney JF, Stewart LA, Ghersi D, Burdett S, Sydes MR. Practical methods for incorporating summary time-to-event data into meta-analysis. Trials. 2007:8:1-16.

25. Debray TPA, Moons KGM, Riley RD. Detecting small-study effects and funnel plot asymmetry in meta-analysis of survival data: a comparison of new and existing tests. Res Synth Methods. 2018 Mar 1:9(1):41-50.

26. Peters JL, Sutton AJ, Jones DR, Abrams KR, Rushton L. Comparison of two methods to detect publication bias in meta-analysis. J Am Med Assoc. 2006 Feb 8;295(6):676-80.

27. Campbell M, McKenzie JE, Sowden A, Katikireddi SV, Brennan SE, Ellis S, et al. Synthesis without meta-analysis (SWiM) in systematic reviews: reporting guideline. BMJ. 2020;368:1-6.

28. Foroutan F, Guyatt G, Zuk V, Vandvik PO, Alba AC, Mustafa R, et al. GRADE guidelines 28: use of GRADE for the assessment of evidence about prognostic factors: rating certainty in identification of groups of patients with different absolute risks. J Clin Epidemiol. 2020;121:62-70.

29. Hoffman JIE, Kaplan S. The incidence of congenital heart disease. J Am Coll Cardiol. 2002;39(12):1890-900

\section{Publisher's Note}

Springer Nature remains neutral with regard to jurisdictional claims in published maps and institutional affiliations.

Ready to submit your research? Choose BMC and benefit from:

- fast, convenient online submission

- thorough peer review by experienced researchers in your field

- rapid publication on acceptance

- support for research data, including large and complex data types

- gold Open Access which fosters wider collaboration and increased citations

- maximum visibility for your research: over $100 \mathrm{M}$ website views per year

At $\mathrm{BMC}$, research is always in progress.

Learn more biomedcentral.com/submissions 